\title{
Estudo comparativo do conhecimento e prática de atividade física de idosos diabéticos tipo 2 e não diabéticos
}

\author{
Comparative study of knowledge and practice of physical activity \\ for elderly diabetics type 2 and non diabetics
}

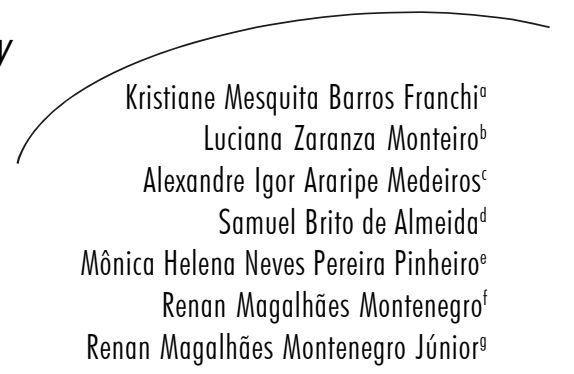

\section{Resumo}

Objetivo: caracterizar o conhecimento e a prática de atividades físicas em idosos diabéticos tipo 2, assistidos em um ambulatório de referência da cidade de Fortaleza, comparando-os com idosos não-diabéticos. Métodos: O estudo, do tipo transversal, envolveu 44 idosos com Diabetes Mellitus tipo 2 (DM2) e 44 sem DM2, avaliados por meio de um questionário sobre prática, recomendações, orientações e conhecimentos de atividades físicas. Os dados foram analisados por meio do teste Qui-quadrado. O nível de significância foi de $\mathrm{p}<0,05$. Resultados: Em relação à prática e a freqüência semanal de alguma atividade física, verificou-se que aproximadamente metade dos participantes dos dois grupos não praticava nenhuma atividade física. Dentre as recomendações recebidas de profissionais da saúde sobre a prática de atividades físicas, $84,1 \%(\mathrm{~N}=37)$ dos idosos diabéticos responderam

Palavras-chave: Idoso. Atividade Motora. Diabetes Mellitus Tipo 2. Estudo Comparativo. Epidemiologia Descritiva. Estudos Transversais. Análise Quantitativa. Fortaleza, CE

Universidade de São Paulo

Faculdade de Medicina de Ribeirão Preto

Ribeirão Preto, SP, Brasil

Correspondência / Correspondence

Luciana Zaranza Monteiro

Rua São Salvador, 328/13

14055-260 - Ribeirão Preto, SP, Brasil

E-mail: lucianazaranza@hotmail.com 


\section{Abstract}

Objective: this study aimedto characterize the knowledge and practice of physical activity in elderly with type 2 diabetes, cared for in a referral outpatient's clinic in Fortaleza city, compared with non-diabetics. Methods: The cross-sectional study comprised 44 elderly people with type 2 Diabetes Mellitus (DM2) and 44 without DM2, who were interviewed through a questionnaire regarding practice, recommendations, guidance and knowledge of physical activities. Data were analyzed using Chi-Squared test. The level of significance was $\mathrm{p}<0.05$. Results: Regarding the practice and weekly frequency of a physical activity, it was verified that approximately half of participants in both groups did not practice any physical activity. Concerning the recommendation received from health professionals on physical activity, $84.1 \%(\mathrm{~N}=37)$ of dibetic elderly replied that they had received this advice, whereas only $58.7 \%(\mathrm{~N}=27)$ of the non-diabetic ones said they had been

Key words: Aged. Motor Activity. Diabetes Mellitus Type 2. Comparative Study. Epidemiology. Descriptive. CrossSectional Studies. Quantitative Analysis. Fortaleza City, CE

\section{INTRODUÇÃ̃O}

Entende-se por idoso ou pessoa da terceira idade, o indivíduo com mais de 60 anos de idade. A população de idosos vem aumentando consideravelmente, o que tem sido atribuído a um aumento da expectativa de vida, a um melhor controle de doenças infecto-contagiosas (imunização) e crônico-degenerativas. ${ }^{1}$

Segundo dados da Organização Mundial da Saúde, ${ }^{2}$ existiam 390 milhões de pessoas acima de 65 anos em 1998 e estima-se que em 2025 essa população será o dobro. Em muitos países em desenvolvimento, especialmente na América Latina e Ásia, é esperado um aumento de $300 \%$ na população idosa, que chegará a dois bilhões de pessoas acima de 60 anos até 2025 .
Esse aumento da população idosa gera necessidades de mudanças na estrutura social para que essas pessoas, ao terem suas vidas prolongadas, não fiquem distantes de um espaço social, em relativa alienação, inatividade, incapacidade física, dependência - conseqüentemente, sem qualidade de vida.

O Diabetes Mellitus tipo 2 (DM2) está entre as doenças crônicas mais prevalentes na população idosa. Quanto mais a população envelhece maior, é a proporção com DM2 e, conseqüentemente, suas complicações se expandem. Além das complicações mais comuns, como as agudas - cetose e cetoacidose diabética, coma diabético e hipoglicemia - e as crônicas - retinopatia, nefropatia, neuropatia e macroangiopatia diabética -, o diabetes tem sido associado como risco alto no 
declínio físico e cognitivo, traumas por quedas, fraturas e depressão. ${ }^{3}$ A presença de neuropatia autonômica no idoso diabético pode limitar a capacidade de realizar atividade física e aumentar o risco de um evento adverso cardiovascular durante a atividade física. ${ }^{4}$

Estudos epidemiológicos indicam que o DM deverá acometer mais de 200.000.000 de pessoas em todo o mundo até o ano 2010 , o que representa o dobro dos valores observados em 1994, ocorrendo predomínio incremental em países da Ásia, da África e da América do Sul. Dentre os fatores que explicariam esses dados, destacam-se o aumento das faixas populacionais mais idosas, como conseqüência da maior expectativa de vida da população e por mudanças dos padrões alimentares e do estilo de vida. ${ }^{3}$

O controle do DM deve se basear em quatro aspectos: a alimentação, a medicação (insulina e/ou antidiabéticos orais), a educação e os exercícios físicos. ${ }^{5}$ Os exercícios físicos têm um efeito depressor sobre os níveis de glicose sangüínea, pois há maior entrada de glicose nos músculos esqueléticos, diminuindo assim a glicemia em indivíduos com DM2, favorecendo, portanto, uma melhora em seu estado.

A American Diabetes Association ${ }^{6}$ reforça que os efeitos benéficos do exercício físico são substanciais, e estudos recentes reforçam a importância de programas de atividades físicas no longo prazo para o tratamento e prevenção desta anormalidade metabólica e suas complicações. Os efeitos benéficos são: diminuição das concentrações de glicose san- güínea antes e após o exercício, melhora do controle glicêmico, diminuição de medicamentos orais ou insulina, melhora da sensibilidade à insulina e do condicionamento cardiovascular e conseqüente diminuição dos fatores de risco cardiovascular.

Mesmo com a importância da prática de atividades físicas como prevenção e promoção da saúde dos idosos com DM2, não encontramos estudos de abrangência local e até mesmo nacional que mostrassem o conhecimento e a prática de atividades físicas desta população, para que pudessem fundamentar a construção de programas educacionais de atividades físicas, campanhas, políticas públicas, treinamento de profissionais da saúde e criação de espaços apropriados voltados para a promoção da saúde através de uma vida mais ativa.

O presente estudo visou a comparar o conhecimento e a prática de atividade física de idosos diabéticos tipo 2 e não-diabéticos.

\section{METODOLOGIA}

Trata-se de estudo de abordagem quantitativa, descritivo e transversal. Os idosos diabéticos foram avaliados no Ambulatório de Diabetes do Adulto, do Serviço de Endocrinologia e Diabetes, do Hospital Universitário Walter Cantídio, da Faculdade de Medicina da Universidade Federal do Ceará (HUWCFAMED-UFC) e os não-diabéticos, no Centro de Lazer Patrolino Oliveira da Prefeitura de Fortaleza-CE. 
Dos 80 idosos diabéticos acompanhados no ambulatório no dia do estudo, 44 concordaram em participar espontaneamente. $\mathrm{O}$ Centro de Lazer é constituído por 72 idosos, sendo que 44 idosos não tinham DM2. Foram incluídos no estudo idosos com idade entre 60 a 80 anos, sem comprometimento de memória que prejudicasse as informações a serem investigadas, com participação voluntária e com assinatura ou impressão digital em termo de consentimento livre e esclarecido, após orientação quanto aos objetivos e procedimentos da pesquisa.

Para a caracterização do grupo dos diabéticos, foi realizada uma medida da glicohemoglobina $\left(\mathrm{Hb}_{\mathrm{AlC}}\right)$, de amostra sanguínea coletada em um período não superior a dois meses do momento da avaliação e uma medida de glicemia capilar em jejum.

A determinação da glico-hemoglobina $\left(\mathrm{Hb}_{\mathrm{A} 1 \mathrm{c}}\right)$ foi realizada no Laboratório Central, do HUWC-FAMED-UFC, em um mesmo ensaio, utilizando-se Kit da In Vitro Diagnóstica Ltda. De acordo com a Sociedade Brasileira de Diabetes, ${ }^{7}$ os valores de referência de normalidade são $\leq 7 \%$.

No grupo dos não-diabéticos, foi utilizada uma medida de glicemia capilar aleatória, utilizando-se como critério de normalidade valores de glicemia $\leq 140 \mathrm{mg} /$ dl duas horas após sobrecarga de glicose, segundo a Sociedade Brasileira de Diabetes. ${ }^{7}$ A coleta foi realizada em todos os indivíduos no período pós-prandial da tarde. Para a glicemia capilar em jejum e pós-prandial, foi utilizado um aparelho deglicosimetria portátil, One Touch Ultra ${ }^{\circledR}$ (Johnson \& Johnson).
A medida da glico-hemoglobina $\left(\mathrm{Hb}_{\mathrm{Alc}}\right)$ foi realizada somente nos idosos diabéticos, pois, de acordo com a American Diabetes Association, ${ }^{8}$ a dosagem da glico-hemoglobi$\mathrm{na}\left(\mathrm{Hb}_{\mathrm{Alc}}\right)$ tem papel fundamental na monitorização do controle glicêmico em pacientes diabéticos, pois fornece informações acerca do índice retrospectivo da glicose plasmática, sendo a melhor opção para a avaliação do controle glicêmico em médio e longo prazo. Esse exame deve ser realizado rotineiramente em todos os pacientes com Diabetes Mellitus, para documentar o grau de controle glicêmico. ${ }^{89}$ Por esse exame ser fundamental na avaliação do controle glicêmico dos diabéticos, aqueles que não apresentaram essa enfermidade não foram submetidos a esse exame, e sim ao teste de glicemia capilar aleatória.

Uma vez selecionados, os participantes foram convidados a responder um questionário não validado elaborado pelos pesquisadores do estudo para esta população, com a finalidade de avaliar a prática, recomendações/ orientações e conhecimentos de atividades físicas em relação ao idoso e ao diabetes. O questionário foi dividido em três partes: Parte I - práticas de atividades físicas: (Você realiza alguma atividade física? Quais as atividades físicas que você pratica? Há quanto tempo (meses), freqüência e minutos por dia que realiza essas atividades?); Parte II - recomendações/orientações sobre atividades físicas: (Já foi recomendada a realização de atividade física por algum profissional em unidades de saúde? Você já foi orientado sobre atividade física por profissionais de saúde? Qual profissional orientou? Quais a formas de orien- 
tações sobre a prática de atividade física que você já recebeu? Quais as atividades físicas the foram recomendadas?); Parte III - conhecimento sobre atividades físicas: (1) - em relação ao idoso: Você acha que a atividade física pode beneficiar o idoso? Você acha que a atividade física deve ser sempre evitada na sua idade? (2) - em relação ao diabetes: você acha que a atividade física pode beneficiar uma pessoa com diabetes? Você acha que a atividade física deve ser sempre evitada pelo portador de diabetes? (3) - quanto aos parâmetros recomendados para a prática de atividades físicas: qual intensidade correta para o exercício físico? Qual é o tempo mínimo para a prática de atividades físicas? Você acha que o exercício aeróbio é o ideal para a melhoria da saúde?

Os dados foram processados no programa GraphPad Prism. ${ }^{10}$ Foram calculados a média e desvio padrão da média (DP), e realizado o teste Qui-quadrado. O nível de significância foi de $\mathrm{p}<0,05$.

Foram respeitados os princípios éticos e legais, de acordo com as recomendações da Resolução CONEP n. ${ }^{\circ}$ 196/96. ${ }^{11}$ O projeto foi submetido e aprovado pelo Comitê de Ética da Universidade de Fortaleza - UNIFOR (Parecer no 129/2004).

\section{RESULTADOS}

Foram avaliados 44 idosos com DM2, dos quais $70,5 \%(\mathrm{n}=31)$ eram do sexo feminino e $29,5 \%(n=13)$ do sexo masculino. Embora não haja diferença de gênero em termos de prevalência do diabetes, a amostra avaliada teve a predominância de idosos do sexo feminino, o que pode refletir a maior longevidade das mulheres em relação aos homens. Segundo Coelho Filho, ${ }^{12}$ este fenômeno se atribui à menor exposição a determinados fatores de risco, principalmente no trabalho, menor prevalência de tabagismo e uso de álcool, diferenças quanto à atitude em relação a doenças e incapacidades e maior freqüência do sexo feminino em atendimentos de saúde.

A média de idade foi de $68,3 \pm 5,4$ anos. O tempo médio de diagnóstico do diabetes foi de 11,2 \pm 7,3 anos. A média da glicemia capilar foi de 138,2 $\pm 18,3 \mathrm{mg} / \mathrm{dl}$. O perfil metabólico foi de $9,7 \pm 2,3 \%$, onde, segundo a Sociedade Brasileira de Diabetes, ${ }^{7}$ o normal é $\leq 7 \%$.

Dentre os idosos não-diabéticos, $84,1 \%$ $(\mathrm{n}=37)$ eram do sexo feminino e 15,9\% $(\mathrm{n}=7)$ do sexo masculino. A média de idade foi de $72,8 \pm 6,2$ anos e a média da glicemia capilar foi de $110,5 \pm 16,1 \mathrm{mg} / \mathrm{dl}$.

Em relação à prática de atividades físicas pelos idosos diabéticos, verificou-se que $52,3 \%(\mathrm{n}=23)$ as praticavam mais do que três vezes por semana, $2,3 \%(\mathrm{n}=1)$ até duas vezes por semana e $45,4 \%(n=20)$ não praticavam nenhum tipo de atividade física (figura 1). Talvez estes resultados demonstrem que o idoso, quando acredita na importância de algo para sua saúde, principalmente quando está doente, adquire de fato a prática esportiva prescrita. 


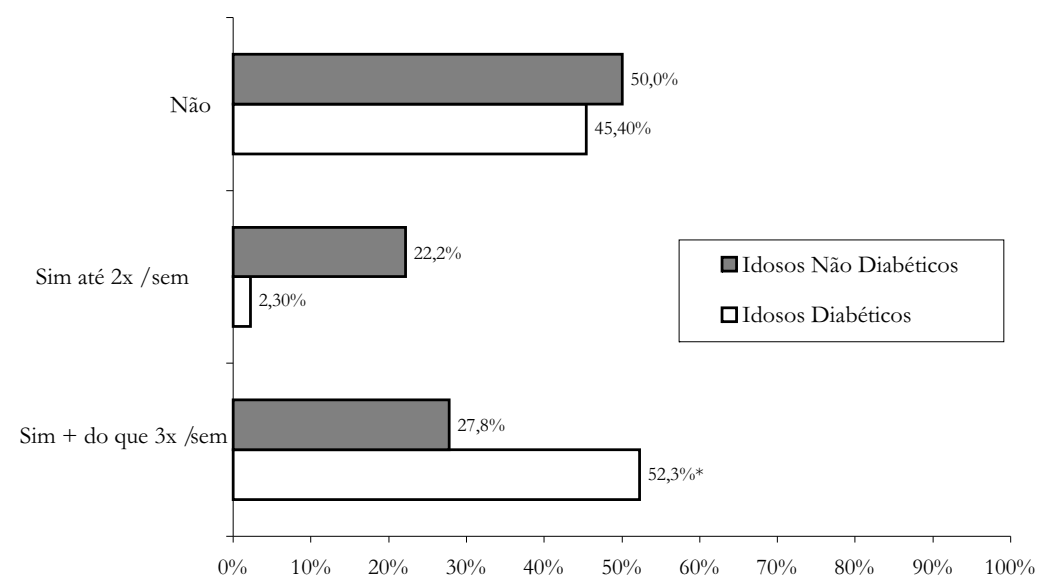

Figura 1 - Prática e freqüência semanal de atividade física dos idosos diabéticos e não-diabéticos $\left({ }^{*} \mathrm{p}<0,05\right.$ e $\left.x^{2}=22,28\right)$.

Dentre as atividades físicas praticadas, as mais freqüentes foram a caminhada, com $66,7 \%(\mathrm{n}=16)$, seguida de alongamento $12,6 \%$ $(\mathrm{n}=3)$, hidroginástica $8,3 \%(\mathrm{n}=2)$, exercícios de força $8,3 \%(n=2)$ e ciclismo $4,1 \%(n=1)$. O tempo médio da prática de atividades físicas foi de 25,4 $\pm 24,8$ meses, com duração em média de $40 \pm 14,8$ minutos e a freqüência de $4,6 \pm 1,2$ vezes por semana.

No grupo de idosos não diabéticos, pôdese observar que $52,3 \%(\mathrm{n}=23)$ não praticavam atividades físicas regulares, $20,4 \%(n=9)$ as praticavam até duas vezes por semana e $27,3 \%(n=12)$ mais de três vezes por semana (Figura 1). Dentre as atividades físicas, a mais praticada foi a caminhada, $\operatorname{com} 66,7 \%(n=14)$, $19 \%(\mathrm{n}=4)$ relataram a prática de alongamento e a dança com $14,3 \%(n=3)$. O tempo médio da prática de atividades físicas foi de 37,1 meses, com duração em média de 41,7 minutos e a freqüência de 3,7 vezes por semana. Observamos que nos dois grupos a caminhada foi a atividade física mais pratica- da, o que se deve ao fato que esta é a forma de exercício mais básica, acessível e ideal para o idoso. Sua prática resulta em importantes benefícios para a saúde, pois é uma atividade física de baixo impacto, que reduz a incidência de lesões, além de ser realizada em diferentes intensidades e em qualquer local, contribuindo para aumentar o contato social, principalmente com grupos de risco de isolamento, depressão e demência. ${ }^{5}$

Dentre as recomendações recebidas pelos profissionais da saúde sobre a realização de atividades físicas, $84,1 \%(n=37)$ dos idosos diabéticos responderam que as tinham recebido, enquanto que entre os idosos não-diabéticos $58,7 \%(\mathrm{n}=27)$ afirmaram que essas recomendações foram dadas, havendo diferença estatisticamente significativa (tabela 1). Observou-se também, na tabela 1 , que $30,9 \%$ dos idosos diabéticos foram orientados sobre como realizar essas atividades físicas. 
Tabela 1 - Recomendações e orientações dos profissionais de saúde quanto à realização de atividades física. Fortaleza, CE, 2007.

\begin{tabular}{lcc}
\hline & Idosos diabéticos & Idosos não-diabéticos \\
\hline Receberam recomendações & $84,1 \% *$ & $58,7 \%$ \\
Receberam orientações & $30,9 \%$ & $30,4 \%$ \\
\hline
\end{tabular}

$\left(* \mathrm{p}<0,05\right.$ e $\left.\mathrm{x}^{2}=14,58\right)$.

A tabela 2 demonstra que o médico foi o profissional de saúde que mais recomendava a pratica de atividades físicas para os idosos diabéticos e não-diabéticos.

Tabela 2 - Profissionais de saúde que recomendavam atividades físicas (AF), Fortaleza, CE, 2007.

\begin{tabular}{lcc}
\hline \multicolumn{1}{c}{ Recomendações de AF } & Idosos Diabéticos & Idosos Não-Diabéticos \\
\hline Pelo(a) médico(a) & $92,3 \%$ & $76,9 \%$ \\
Pelo(a) enfermeiro(a) & $10,3 \%$ & $3,8 \%$ \\
Pelo(a) nutricionista(a) & $7,7 \%$ & 0 \\
Pelo(a) educador(a) físico(a) & $2,6 \%$ & $7,7 \%$ \\
Por bombeiros & - & $3,8 \%$ \\
Por não-profissionais (praticantes de AF) & $2,6 \%$ & $7,7 \%$ \\
\hline
\end{tabular}

Quanto às formas de orientações recebidas, observou-se na tabela 3 que as orientações verbais foram as que predominaram em ambos os grupos, sendo, contudo, mais freqüente no grupo de idosos diabéticos $82,1 \%(\mathrm{n}=33)$ do que dos não-diabéticos 41,7\% (n=18). Acredita-se que as orientações verbais, dadas na maioria pelos médicos, se dão em geral através de informações fornecidas durante as consultas médicas. Sabe-se que a abordagem biomédica represen- ta apenas uma das cinco formas de intervenção social no cenário da saúde pública, sendo as outras: a abordagem da mudança de comportamento, a abordagem educativa, a abordagem centralizada no cliente e a abordagem à sociedade. ${ }^{4}$ Isto pode demonstrar, portanto, a necessidade de reestruturação das ações em educação em saúde, pois poderiam ser introduzidas boas estratégias de informação e educação para orientar as pessoas. 
Tabela 3 - Formas de orientações recebidas sobre a prática de atividades. Fortaleza, CE, 2007.

\begin{tabular}{lcc}
\hline Formas de orientação recebidas & Idosos diabéticos & Idosos não-diabéticos \\
\hline Orientações verbais & $82,1 \% *$ & $41,7 \%$ \\
Folhetos explicativos & $23,1 \%$ & $36,1 \%$ \\
Vídeos & - & $8,4 \%$ \\
Reuniões de grupo & - & $13,8 \%$ \\
\hline
\end{tabular}

$\left({ }^{*} \mathrm{p}<0,05\right.$ e $\left.\mathrm{x}^{2}=21,04\right)$.

Verificou-se que a caminhada foi a atividade física mais recomendada para ambos os grupos. Entretanto, no grupo dos diabéticos $87,2 \%(\mathrm{n}=34)$ tal recomendação foi mais evidente do que para os não-diabéticos $62,9 \%$ $(n=22)$, sendo esta diferença estatisticamente significativa (tabela 4). Contudo, menos da metade dos avaliados havia recebido a orientações sobre como fazer essa caminhada, tipo de calçado, intensidade, contra-indicações e cuidados necessários. Isto pode sugerir a ne- cessidade da melhora nas informações e abrangência de ações no ambiente de atendimento à saúde, ainda muito centrado na atuação do médico isoladamente. Seria interessante a inclusão do profissional de Educação Física, que poderia, junto à equipe multidisciplinar, orientar tanto a equipe de saúde como os pacientes, planejar e executar ações que contribuíssem para a mudança de estilos de vida, para os progressos em relação à qualidade de vida.

Tabela 4 - Tipos de atividades físicas (AF) recomendadas. Fortaleza, CE, 2007

\begin{tabular}{lcc}
\hline AF recomendadas & Idosos diabéticos & Idosos não-diabéticos \\
\hline Caminhada & $87,2 \% *$ & $62,9 \%$ \\
Hidroginástica & $5,1 \%$ & $2,8 \%$ \\
Alongamento & $5,1 \%$ & $20 \%$ \\
Exercícios de força & $2,6 \%$ & $11,4 \%$ \\
Yoga & - & $3,6 \%$ \\
\hline
\end{tabular}

$\left({ }^{*} \mathrm{p}<0,05\right.$ e $\left.\mathrm{x}^{2}=9,56\right)$. 
Avaliando o conhecimento dos idosos diabéticos e não-diabéticos sobre os benefícios da prática de atividades físicas para o idoso, observou-se que todos os diabéticos afirmaram que a atividade física pode beneficiar o idoso e não deve ser evitada. No grupo dos não-diabéticos, 4,5\% $(\mathrm{n}=2)$ responderam que não consideravam a atividade física benéfica, devendo assim, ser evitada.

Em relação à opinião dos indivíduos a respeito dos benefícios da atividade física para os portadores de diabetes, $81,8 \%(\mathrm{n}=36)$ dos idosos diabéticos afirmaram que a atividade física pode beneficiá-los, e 18,2\% ( $\mathrm{n}=8)$ responderam que não sabiam, diferindo estatisticamente dos idosos não-diabéticos, dos quais $72,8 \%(\mathrm{n}=32)$ não souberam responder e $27,2 \%(n=12)$ afirmaram que a atividade física pode beneficiar o portador de diabetes.

Comparando os grupos quanto aos parâmetros recomendados para a prática de atividades físicas, observou-se que os idosos diabéticos apresentaram conhecimento melhor em relação ao tipo de intensidade recomen- dada para o exercício físico, comparados aos não-diabéticos (tabela 5). O American College of Sports Medicine ${ }^{13}$ recomenda a intensidade moderada de exercícios aeróbios para as pessoas idosas, pois esta mostra significativos efeitos fisiológicos após um período de treino. Já em relação ao tempo mínimo ideal para a prática de atividades físicas (30 minutos por dia), o grupo de idosos não-diabéticos respondeu corretamente, se comparados aos diabéticos.

Baseando-se nas recomendações do American College of Sports Medicine, ${ }^{13}$ a saúde acontece quando o indivíduo realiza um exercício regular de moderada intensidade por no mínimo 30 minutos na maioria dos dias da semana. Entretanto, os idosos diabéticos apresentaram maior número de respostas corretas, não achando que o exercício aeróbio é o ideal para a melhoria da saúde (tabela 5). Observamos que os idosos diabéticos apresentaram melhor conhecimento sobre esses parâmetros, o que pode ocorrer devido às informações fornecidas durante as consultas médicas para os diabéticos.

Tabela 5 - Conhecimento dos idosos diabéticos e dos idosos não-diabéticos quanto aos parâmetros recomendados para a prática de atividades físicas. Fortaleza, CE, 2007.

\begin{tabular}{|c|c|c|c|c|c|c|}
\hline \multirow[b]{2}{*}{ Pergunta formulada } & \multicolumn{3}{|c|}{ Idosos diabéticos } & \multicolumn{3}{|c|}{ Idosos não-diabéticos } \\
\hline & $\begin{array}{l}\text { Sim } \\
(\%)\end{array}$ & $\begin{array}{l}\text { Não } \\
(\%)\end{array}$ & $\begin{array}{c}\text { Não sabe } \\
(\%)\end{array}$ & $\begin{array}{l}\text { Sim } \\
(\%)\end{array}$ & $\begin{array}{l}\text { Não } \\
(\%)\end{array}$ & $\begin{array}{c}\text { Não Sabe } \\
(\%)\end{array}$ \\
\hline Intensidade máxima de atividade física & 4,5 & $65,9 *$ & 29,6 & 34 & 45,5 & 20,5 \\
\hline Intensidade forte de atividade física & 11,4 & 59,1 & 29,5 & 31,8 & 50 & 18,2 \\
\hline Intensidade moderada de atividade física & $93,2^{*}$ & 4,5 & 2,3 & 34,1 & 43,2 & 22,7 \\
\hline Tempo mínimo ideal de atividade física (30 min) & 63,6 & 11,4 & 25 & $72,7^{*}$ & 2,3 & 25 \\
\hline Tipo de exercício (aeróbio) & 15,9 & $65,9 *$ & 18,2 & 29,5 & 20,5 & 50 \\
\hline
\end{tabular}

Respostas esperadas, em negrito $\left({ }^{*} \mathrm{p}<0,05\right)$ 


\section{DISCUSSÃO}

No presente estudo, observou-se um número maior de participantes do sexo feminino, o que comprova o fenômeno de feminização do processo do envelhecimento, conforme apontam Freire e Tavares. ${ }^{14} \mathrm{~A}$ feminização vem ocorrendo desde o final da década de 80, mas há muito tempo tem sido superior, no país, o número absoluto de mulheres idosas, quando comparado ao de homens idosos. Segundo dados da última Pesquisa Nacional de Amostra por Domicílios, ${ }^{15}$ entre os idosos o percentual feminino vem aumentando lentamente: em 1981, com 52,6\%; em 1999, com 55,3\%; e em 2004, com $56,1 \%$ de mulheres idosas.

Em relação à faixa etária dos participantes, notou-se que os idosos diabéticos apresentaram idade acima de 68 anos e os nãodiabéticos, 72 anos. Segundo Freire e Tavares, ${ }^{14} \mathrm{em}$ idades mais avançadas acentuam-se as limitações, levando a uma maior dependência nas atividades cotidianas.

Atualmente está comprovado que quanto mais ativa é uma pessoa, menos limitações físicas ela tem. ${ }^{13}$ No presente estudo, verificouse que quando comparada a prática de atividades físicas dos idosos diabéticos com os não-diabéticos, os não-diabéticos (52,3\%) não se exercitavam regularmente. De acordo com Chaimowicz, ${ }^{16}$ a prática regular de atividade física, mesmo se iniciada após os 65 anos de idade, contribui para uma maior longevidade, melhora da capacidade fisiológica, redução do número de medicamentos prescritos, benefícios psicológicos, como melhora da auto-estima, entre outros.
Estudos vêm evidenciando a atividade física como importante requisito para minimizar a degeneração provocada pelo envelhecimento, permitindo ao idoso manter uma qualidade de vida ativa. Segundo Okuma, ${ }^{17}$ evidências mostraram que mais da metade do declínio da capacidade física dos idosos se deve à depressão, inatividade e expectativa de enfermidades, e que a atividade física regular e sistêmica aumenta ou mantém a aptidão física da população idosa, tendo o potencial de melhorar o bem-estar funcional e, assim, diminuir a taxa de morbidade e mortalidade dessa população.

Segundo Mota, ${ }^{18}$ um programa de exercícios deve promover a melhoria da capacidade física do indivíduo, intervindo sobre os efeitos deletérios resultantes do processo de envelhecimento, conseguindo maximizar o contato social dos sujeitos e procurando reduzir os problemas psicológicos, como ansiedade e depressão, características desse grupo populacional.

Quanto às orientações dadas aos idosos diabéticos sobre como realizar as atividades físicas, somente $30,9 \%$ as recebiam, o que comprova o estudo de Guimarães e Takayanagui. ${ }^{19}$ Estes, ao realizarem levantamento das principais orientações recebidas por um grupo de pacientes diabéticos no momento do diagnóstico, em uma unidade básica e distrital de saúde, concluíram que $82,8 \%$ dos pacientes mencionaram ter sido orientados apenas para o tratamento com dieta e/ou medicação - isto é, sem nenhuma orientação para a prática de atividades físicas. Isto mostra a necessidade de as equipes de saúde reverem suas 
práticas de educação em saúde relacionadas ao tratamento dos DM2.

O estudo mostrou que a caminhada foi a atividade física mais recomendada para ambos os grupos, mas os profissionais de saúde que a recomendavam não sabiam prescrever como deveriam realizá-la, assim como o tipo de calçado, intensidade, contra-indicações e cuidados necessários. Em trabalho realizado por Pollock, ${ }^{20}$ sobre a prescrição de exercícios para idosos, observou-se maior incidência de lesões para os sujeitos de 70 a 79 anos que praticavam corrida, do que para os que praticavam somente a caminhada.

Segundo a American Diabetes Association, ${ }^{6}$ pacientes diabéticos com perda da sensibilidade estão contra-indicados a praticar caminhada, sendo mais recomendados o ciclismo e a natação. Sendo assim, é necessário adequar a intensidade dos exercícios e acrescentar cuidados importantes e específicos para pacientes diabéticos.

A maioria dos idosos diabéticos recebia dos médicos recomendações para praticarem a caminhada como atividade física regular. Segundo Guimarães, ${ }^{21}$ hoje se valoriza, merecidamente, a atuação médica que conseguiu evitar que o paciente morresse na fase aguda da doença, mas ignora-se a necessidade de uma abordagem multidisciplinar mais abrangente, na qual se trabalhe com a Educação em Saúde através de ações interdisciplinares, integrando vários conhecimentos, possibilitando mudança de comportamento da clientela para o alcance de melhor nível de saúde.
Os idosos diabéticos afirmaram que o exercício aeróbio não é o ideal para a melhoria da saúde. De acordo com Winett e Carpinelli, ${ }^{22}$ os exercícios de força, também conhecidos como resistidos, geralmente realizados com pesos, têm sido recomendados como ideais para pessoas idosas, pois aumentam a massa óssea e a muscular, ajudam a reduzir o tecido adiposo e aprimoram a força muscular, flexibilidade e a resistência.

\section{CONCLUSÃO}

A partir do exposto, conclui-se que os idosos diabéticos mostraram melhor conhecimento e prática regular de atividade física do que os idosos não-diabéticos.

Para isso, propomos ações nas quais o idoso adquira conhecimentos para a descoberta de seus potenciais e limites físicos e motores, com conseqüentes descobertas emocionais, sociais e cognitivas. Deverá ser uma proposta puramente educacional, que objetivará modificar comportamentos para a prática sistemática de atividades físicas, valorizando-a como meio de manutenção da saúde.

\section{NOTAS}

Educadora Física, Mestre em Educação em Saúde, Departamento de Educação Física, Universidade de Fortaleza. Atuou na pesquisa, metodologia, na concepção e na redação final. E-mail: kri70@unifor.br

b Educadora Física, Mestre e Doutoranda em Clínica Médica, Faculdade de Medicina de Ribeirão Preto - Universidade de São Paulo. Atuou na pesquisa, metodologia, na redação final. E-mail:_lucianazaranza@hotmail.com 
Educador Físico, Especialista em Treinamento Desportivo pela Universidade Estadual do Ceará . Atuou na pesquisa (coleta dos dados). E-mail: alexandreararipe@hotmail.com

d Educador Físico pela Universidade de Fortaleza. Atuou na pesquisa (coleta dos dados). E-mail: sb_ef@hotmail.com

e Educadora Física, Mestre, Coordenadora do Curso de Especialização em Fisiologia do Exercício, Atividade Física, Nutrição e Saúde - Universidade de Fortaleza. Atuou na análise estatística e metodologia. E-mail: monicahelenaneves@hotmail.com

\section{REFERÊNCIAS}

1. Almeida DT, Leitão GCM, Silva LF. Qualidade de vida e percepção do envelhecimento sob a ótica do idoso. Revista do Centro de Ciências da Saúde Unifor 2000; 12 (12): 27-33.

2. World Health Organization - WHO. Noncommunicable Disease Prevention and Health Promotion. Physical Activity for various population groups - Ageing Population. [acesso em:2004 fev. 26] Disponível em: URL: http://www.who.int/ inf-fs/en/fact135.html

3. Torquato MTCG, Montenegro Jr. RM, Foss MC. Diabetes Mellitus: epidemiologia, conceito, classificação, diagnóstico, tratamento e complicações. Anais do Curso de Atualização em Diabetes para Profissionais de Saúde; 1999; Ribeirão Preto, SP. Ribeirão Preto; 1999. p.4-41.

4. Candeias NMF. Conceitos de educação e de promoção em saúde: mudanças individuais e mudanças organizacionais. Rev Saúde Pública 1997; 31(2): 209-13.

5. Steiner G, Lawrence PA. Educando o paciente diabético. São Paulo: Andrei; 1992.

6. American Diabetes Association. Atividade física / exercícios e diabetes. Diabetes Care 2004; 3: 127-31.

7. Sociedade Brasileira de Diabetes. Tratamento e acompanhamento do Diabetes Mellitus. Rio de Janeiro: Diagraphic; 2007. p.168.
Médico Endocrinologista, Professor Adjunto do Departamento de Medicina Clínica, Faculdade de Medicina Universidade Federal do Ceará. Atuou na pesquisa e na redação final. E-mail:_renanmontenegro@terra.com.br

g Médico Endocrinologista, Professor Adjunto do Departamento de Saúde Comunitária da Faculdade de Medicina - Universidade Federal do Ceará. Atuou na pesquisa, metodologia, na redação final. E-mail: renanjr@ufc.br

8. American Diabetes Association. Clinical practice recommendations. report of the expert committee on the diagnosis and classification of Diabetes Mellitus. Diabetes Care 2003; 26 (suppl. 1): S5-20.

9. Sacks DB, et al. Guidelines and recommendations for laboratory analysis in the diagnosis and management of Diabetes Mellitus. Clin Chem 2002; 48: 436-72.

10. GraphPad Instat versão 3.0 para Windows 95, Copyright 1999. GraphPad Software Inc, San Diego California USA. Disponível em: URL: http:/ / www.graphpad.com

11. Brasil. Ministério da Saúde. Conselho Nacional de Saúde. Normas de pesquisa envolvendo seres humanos. Res. CNS 196/ 96. Bioética, 1996; 4 (Suppl):15-25.

12. Coelho Filho JM, Ramos LR. Epidemiologia do envelhecimento no Nordeste do Brasil: resultados de inquérito domiciliar. Rev Saúde Pública 1999; 33 (5): 445-53.

13. American College of Sports Medicine. Position stand on exercise and physical activity for older adults. Med Sci Sports Exerc 1998; 30: 992-1008.

14. Freire Junior RC, Tavares MFL. A promoção de Saúde nas instituições de longa permanência: uma reflexão sobre o processo de envelhecimento no Brasil. Revista brasileira de geriatria e gerontologia 2006; 9 (1): 83-92. 
15. Fundação Instituto Brasileiro de Geografia e Estatística (2004). Pesquisa Nacional de Amostra por Domicílio no Brasil (PNAD) [acesso 2006 jul 20]. Disponível em URL: http://www.ibge.gov.br

16. Chaimowicz F. A saúde dos idosos brasileiros às vésperas do século XXI: problemas, projeções e alternativas. Rev Saúde Pública 1997; 31 (1): 184-202.

17. Okuma SS. O idoso e a atividade física. 1. ed. Campinas: Papirus; 1998.

18. Mota J. Envelhecimento e exercício - atividade física e qualidade de vida na população idosa. In: Barbanti VJ, et al. Esporte e atividade física: interação entre rendimento e saúde. 1.ed. São Paulo: Manole; 2002.
19. Guimarães FPM, Takayanagui AMM. Orientações recebidas do serviço de saúde por pacientes para o tratamento do portador de Diabetes Mellitus tipo 2. Rev Nutr , 2002; 15 (1): 37-44.

20. Pollock ML. Exercise prescriptions for the elderly. American Academy of Physical Education papers 1989; 22: 163-74.

21. Guimarães RM. Os compromissos da Geriatria. In: Guimarães RM; Cunha UG. Sinais e sintomas em Geriatria. São Paulo: Atheneu; 2004.

22. Winett RA, Carpinelli RN. Potential Health - related benefits of resistance training. Prev Med 2001; 33: 503-13. 
1 